\title{
Degree of fusiform dilatation of the proximal descending aorta in type B acute aortic dissection can predict late aortic events
}

Akira Marui, MD, PhD, ${ }^{\text {a,b }}$ Takaaki Mochizuki, MD, PhD, ${ }^{a}$ Tadaaki Koyama, MD, PhD, ${ }^{c}$ and Norimasa Mitsui, MD, $\mathrm{PhD}^{\mathrm{d}}$

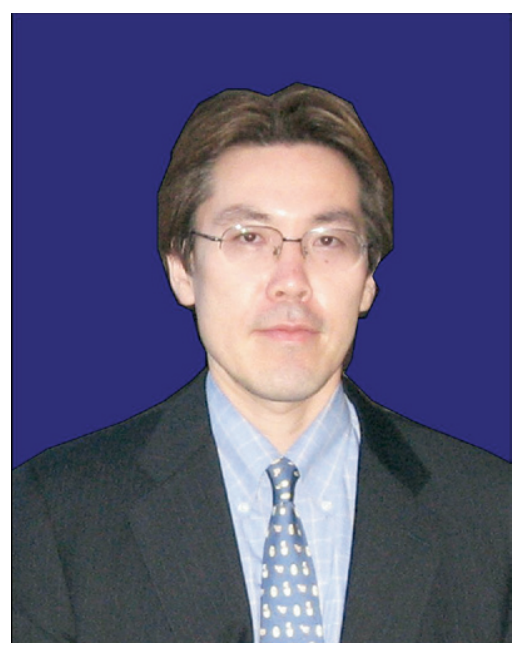

Dr. Marui
From the Department of Cardiovascular Surgery, Akane-Foundation Tsuchiya General Hospital, ${ }^{\text {a }}$ Hiroshima, Japan; Department of Cardiovascular Surgery, Kyoto University Graduate School of Medicine, ${ }^{\mathrm{b}}$ Kyoto, Japan; Department of Cardiovascular Surgery, Shin-Katsushika Hospital, ${ }^{\mathrm{c}}$ Tokyo, Japan; and Department of Thoracic and Cardiovascular Surgery, Hiroshima Prefectural Hospital, ${ }^{\mathrm{d}}$ Hiroshima, Japan.

Received for publication Jan 31, 2007; revisions received July 4, 2007; accepted for publication July 10, 2007.

Address for reprints: Akira Marui, MD, $\mathrm{PhD}$, Department of Cardiovascular Surgery, Kyoto University Graduate School of Medicine, 54 Shogoin-Kawahara, Sakyoku, Kyoto, 606-8507, Japan (E-mail:marui@ kuhp.kyoto-u.ac.jp).

J Thorac Cardiovasc Surg 2007;134:1163-70 $0022-5223 / \$ 32.00$

Copyright @ 2007 by The American Association for Thoracic Surgery

doi:10.1016/j.jtcvs.2007.07.037
Objective: Predicting the risk factors for late aortic events in patients with type B acute aortic dissection without complications may help to determine a therapeutic strategy for this disorder. We investigated whether late aortic events in type B acute aortic dissection can be predicted accurately by an index that expresses the degree of fusiform dilatation of the proximal descending aorta during the acute phase; this index can be calculated as follows: (maximum diameter of the proximal descending aorta)/(diameter of the distal aortic arch + diameter of the descending aorta at the pulmonary artery level).

Methods: Patients with type B acute aortic dissection without complications $(\mathrm{n}=$ 141) were retrospectively analyzed to determine the predictors of late aortic events; these include aortic dilatation, rupture, refractory pain, organ ischemia, rapid aortic enlargement, and rapid enlargement of ulcer-like projections.

Results: The fusiform index in patients with late aortic events (0.59) was higher than that in patients without late aortic events $(0.53, P<.01)$. Patients with a higher fusiform index exhibited aortic dilatation earlier than those with a lower fusiform index. By multivariate analysis, we conclude that the predominant independent predictors of late aortic events were a maximum aortic diameter of $40 \mathrm{~mm}$ or more, a patent false lumen, and a fusiform index of 0.64 or more (hazard ratios, 3.18, 2.64, and 2.73, respectively). The values of actuarial freedom from aortic events for patients with all 3 predictors at 1, 5, and 10 years were $22 \%, 17 \%$, and $8 \%$, respectively, whereas the values in those without these predictors were $97 \%, 94 \%$, and $90 \%$, respectively.

Conclusions: The degree of fusiform dilatation of the proximal descending aorta, a patent false lumen, and a large aortic diameter can be predominant predictors of late aortic events in patients with type B acute aortic dissection. Patients with these predictors should be recommended to undergo early interventions (surgery or stent-graft implantation) or at least be closely followed up during the chronic phase before such events develop.

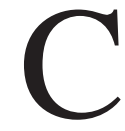

onservative medical treatment is generally the treatment of choice for patients with Stanford type B acute aortic dissection (AAD) without complications (rupture, visceral organ ischemia, or refractory pain) in most institutions, because the natural history of these patients is not severe. ${ }^{1-6}$ However, aortic complications develop during the chronic phase in many patients who receive medical treatment with successful results during the acute phase of this disorder. Therefore, predicting risk factors for the development of late aortic events may help in determining a therapeutic strategy for type B AAD without complications. ${ }^{5-12}$

We reported that a maximum aortic diameter of $40 \mathrm{~mm}$ or more and a patent false lumen during the acute phase were the predominant predictors of late aortic events (aortic diameter $\geq 60 \mathrm{~mm}$, rupture, refractory pain, organ ischemia, rapid aortic 

Abbreviations and Acronyms
$\mathrm{AAD}=$ acute aortic dissection
$\mathrm{CT}=$ computed tomography
FI $=$ fusiform index
$\mathrm{ULP}=$ ulcer-like projection

enlargement $\geq 10 \mathrm{~mm} / \mathrm{y}$, and rapid enlargement of ulcerlike projection [ULPs] $\geq 5 \mathrm{~mm} / \mathrm{y}$ ) in type B AAD without complications. ${ }^{12}$ We suggested that patients with these predictors should undergo early surgery before aortic events occur; however, medical treatment should be continued in patients without these predictors.

A helical flow pattern can be observed in the part of the normal aorta that is distal to the aortic arch (ie, the proximal descending aorta); however, disturbed flow patterns are observed in this part in patients with aneurysms and dissection. ${ }^{13}$ Once morphologic changes have occurred in the aortic wall, this disturbed flow pattern promotes aortic dilatation. ${ }^{14}$ As observed in true aneurysms of the distal aortic arch, an initial morphologic change in the proximal descending aorta (ie, fusiform dilatation) in patients with type B AAD may worsen the disturbed flow pattern and accelerate aneurysmal aortic dilatation. This process is less severe in patients with type B AAD with a lesser degree of initial morphologic change in the aorta.

We hypothesized that the morphologic change in the shape of the proximal descending aorta to a fusiform shape during the acute phase of type B AAD may accelerate the dilatation of the dissected aorta during the chronic phase. To test this hypothesis, we defined a fusiform index (FI) that expresses the degree of fusiform dilatation of the proximal descending aorta. The purpose of the present study was to evaluate the acute-phase predictors of late aortic events, including the FI, and to investigate an optimal therapeutic strategy for type B AAD without serious complications.

\section{Materials and Methods \\ Study Population}

Between January 1988 and March 2004, 153 patients in the acute phase (within 14 days of the onset) of type B AAD were admitted to Tsuchiya General Hospital. Of these, 141 patients without serious complications (rupture, visceral organ ischemia, or refractory pain) were entered into this study as patients who were treated successfully with medical treatment during the acute phase. The remaining 12 patients were excluded from the study because they had either undergone urgent surgery $(\mathrm{n}=7)$ (owing to a ruptured thoracic aorta, $\mathrm{n}=3$; ruptured abdominal aorta, $\mathrm{n}=2$; and visceral organ ischemia, $\mathrm{n}=2)$ or had died of aortic rupture $(\mathrm{n}=$ 3 ) or visceral organ ischemia $(n=2)$. The age of the patients ranged from 32 to 90 years (mean age, 68 years), and 97 patients $(69 \%)$ were male. Patients with AAD and Marfan syndrome were not included (Table 1). We obtained informed consent from all
TABLE 1. Patient characteristics for the entire study group (141 patients)

\begin{tabular}{lc}
\hline Basic characteristics & \\
Sex, male, $\mathrm{n}(\%)$ & $97(69 \%)$ \\
Age, y (mean \pm SD) & $68 \pm 13$ \\
'70 $y, \mathrm{n}(\%)$ & $63(45 \%)$ \\
Hypertension, $\mathrm{n}(\%)$ & $104(74 \%)$ \\
Diabetes mellitus, $\mathrm{n}(\%)$ & $22(16 \%)$ \\
Ischemic heart disease, $\mathrm{n}(\%)$ & $33(23 \%)$ \\
Cerebrovascular disease, $\mathrm{n}(\%)$ & $24(17 \%)$ \\
COPD, $\mathrm{n}(\%)$ & $27(19 \%)$ \\
Hemodialysis, $\mathrm{n}(\%)$ & $11(8 \%)$ \\
LVEF, $\%$ (mean \pm SD) & $62 \pm 15$ \\
$\geq 70 \%, \mathrm{n}(\%)$ & $40(28 \%)$ \\
CT findings during the acute phase & \\
Extent of dissection, $\mathrm{n}(\%)$ & \\
DeBakey Illa & $52(37 \%)$ \\
DeBakey IIlb & $89(62 \%)$ \\
Patency in the false lumen, $\mathrm{n}(\%)$ & \\
Patent & $62(44 \%)$ \\
Closed & $79(56 \%)$ \\
Maximum aortic diameter, $\mathrm{n}(\%)$ & \\
$\geq 40 \mathrm{~mm}$ & $66(47 \%)$ \\
FI (median) & 0.55 \\
$\geq 0.64, \mathrm{n}(\%)$ & $35(25 \%)$ \\
Follow-up variables during the chronic phase & \\
Mean systolic blood pressure & \\
mm Hg (mean \pm SD) & $124 \pm 29$ \\
$\geq 140$ mm Hg, $\mathrm{n}(\%)$ & $20(14 \%)$ \\
\hline
\end{tabular}

$C O P D$, Chronic obstructive pulmonary disease; $\angle V E F$, left ventricular ejection fraction; $C T$, computed tomography; $S D$, standard deviation; $F I$, fusiform index.

patients. The study protocol was reviewed and approved by the institutional review board, and the study complied with the Declaration of Helsinki.

\section{Definition of Fusiform Index}

An index that expresses the degree of fusiform dilatation (the FI) of the proximal descending aorta was defined as follows: $A /(B+C)$ (A, maximum diameter of the proximal descending aorta, B; diameter of the distal aortic arch; and $\mathrm{C}$, diameter of the descending aorta at the level of the origin of the main pulmonary artery) (Figure 1). In cases with retrograde dissection of the aortic arch, we measured the diameter of the aortic arch at the proximal extent of the retrograde dissection and considered this value as the " $\mathrm{B}$ " value.

\section{Diagnosis and Predictive Variables}

The diagnosis of type B AAD was confirmed by contrast-enhanced computed tomography (CT) scanning (from 1988 to 1993 using model TCT-300, Toshiba Co, Tokyo, Japan; from 1994 to 2004 using ProSpeed, GE Yokogawa Medical Systems, Tokyo, Japan) that was performed immediately after urgent admission. We occasionally used digital subtraction angiography to evaluate the visceral arteries to support the CT findings. We obtained the 


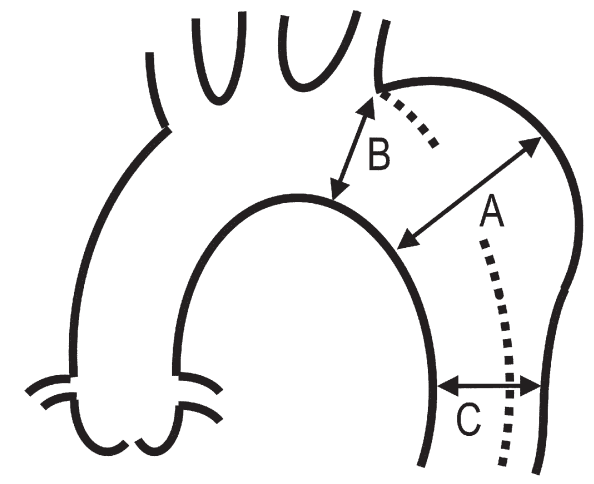

Fusiform index $(F I)=A /(B+C)$

Figure 1. Definition of FI. An index that expresses the degree of fusiform dilatation of the proximal descending aorta was defined as follows: $A /(B+C) . A=$ Maximum diameter of the descending aorta. $B=$ Diameter of the distal aortic arch. $C=$ Diameter of the descending aorta at the level of the origin of the main pulmonary artery.

following data from the examination results: the maximum aortic diameter (measured at any point between the aortic arch and the descending aorta), all aortic diameters that were required for calculating the FI, the thoracoabdominal extent of the dissection extending beyond the diaphragm, and the patency of the false lumen. In patients in whom 3-dimensional CT data were available, each aortic diameter was measured using commercially available software. However, in patients in whom these data were unavailable, we used the same methodology that several studies had used as a surrogate for 3-dimensional CT analysis: ${ }^{10,15}$ In this method, the largest short-axis diameter of the outer contour of the affected segment of the aorta was measured. In the aortic arch, the largest diameter perpendicular to the curvature was measured. The diameters were measured from hardcopy images using direct-reading calipers and corrected for the appropriate scale. These findings were reviewed by an experienced radiologist in a blind manner.

These data on the acute phase, along with the basic characteristics of the patients (sex, age, and presence of a concomitant medical condition such as hypertension, diabetes mellitus, ischemic heart disease, cerebrovascular disease, chronic obstructive pulmonary disease, hemodialysis, and left ventricular ejection fraction), were evaluated as predictive variables of late aortic events during the chronic phase. In addition, the mean systolic blood pressure, which was obtained from serial blood pressure measurements during the chronic phase, was also considered as a predictive variable of late aortic events (Tables 1 and 2).

\section{Follow-up Study and Definitions of Predictive Variables}

All 141 patients received medical treatment during the acute and chronic phases as previously described. ${ }^{12}$ The chronic phase started from day 14 after the onset of AAD. ${ }^{2,4,5}$ The
TABLE 2. Characteristics of patients with and without late aortic events (141 patients)

\begin{tabular}{lrr}
\hline & $\begin{array}{c}\text { Events } \\
(\mathbf{n}=59)\end{array}$ & $\begin{array}{c}\text { No events } \\
\text { (n = 82) }\end{array}$ \\
\hline Basic characteristics & & \\
Sex, male, n (\%) & $42(71 \%)$ & $55(67 \%)$ \\
Age $\geq 70$ y, n (\%) & $24(41 \%)$ & $39(48 \%)$ \\
Hypertension, n (\%) & $47(80 \%)$ & $57(70 \%)$ \\
Diabetes mellitus, n (\%) & $13(22 \%)$ & $9(11 \%)$ \\
Ischemic heart disease, n (\%) & $19(32 \%)$ & $14(17 \%)$ \\
Cerebrovascular disease, n (\%) & $15(25 \%)$ & $9(11 \%)$ \\
COPD, n (\%) & $9(15 \%)$ & $18(22 \%)$ \\
Hemodialysis, n (\%) & $7(12 \%)$ & $4(5 \%)$ \\
LVEF $\geq 70 \%, \mathrm{n}(\%)$ & $21(36 \%)$ & $19(23 \%)$ \\
CT findings during the acute phase & & \\
Extent of dissection, n (\%) & & \\
$\quad$ DeBakey IIla & $23(39 \%)$ & $29(35 \%)$ \\
$\quad$ DeBakey IIlb & $36(61 \%)$ & $53(65 \%)$ \\
Patency in the false lumen, n (\%) & & \\
$\quad$ Patent & $35(59 \%)$ & $27(33 \%)$ \\
$\quad$ Closed & $24(41 \%)$ & $55(67 \%)$ \\
Maximum aortic diameter, n (\%) & & \\
$\quad \geq 40$ mm & $39(66 \%)$ & $27(33 \%)$ \\
$\quad<40$ mm & $20(34 \%)$ & $55(67 \%)$ \\
FI, n (\%) & & \\
$\quad \geq 0.64$ & $23(39 \%)$ & $12(15 \%)$ \\
$\quad<0.64$ & $36(61 \%)$ & $70(85 \%)$ \\
Follow-up variables & & \\
Mean systolic blood pressure, n (\%) & & $11(13 \%)$ \\
$\quad \geq 140$ mm Hg & $9(15 \%)$ & \\
\hline
\end{tabular}

$C O P D$, Chronic obstructive pulmonary disease; $L V E F$, left ventricular ejection fraction; $\mathrm{Fl}$, fusiform index.

criteria used to define late aortic events during the chronic phase were (1) maximum diameter of the dissected aorta of 60 mm or more; (2) rupture of the dissected aorta; (3) refractory pain; (4) visceral organ ischemia; (5) rapid enlargement of the dissected aorta by $10 \mathrm{~mm}$ or more per year; and (6) rapid enlargement of ULPs by $5 \mathrm{~mm}$ or more per year. We obtained the expansion rate of each dissected aorta or ULP by calculating the difference in diameter between the initial measurement and that at the most recent follow-up and dividing this value by the time interval between the 2 measurements. All of the 141 patients with type B AAD who had received medical treatment during the acute phase of this disorder were followed up on an outpatient basis (Table 2). The blood pressure was measured every month. A CT scan was performed every 1 to 34 months (mean, 16.3 months). The CT images of all patients at urgent admission and during follow-up were available.

\section{Statistical Analysis}

All continuous variables are expressed as the mean \pm standard deviation. Comparison of the clinical and radiologic characteristics was performed by the chi-square analysis for categoric variables and by Student $t$ test for continuous variables. To determine whether the FI is a predictive factor, we divided the patients into 
(A)

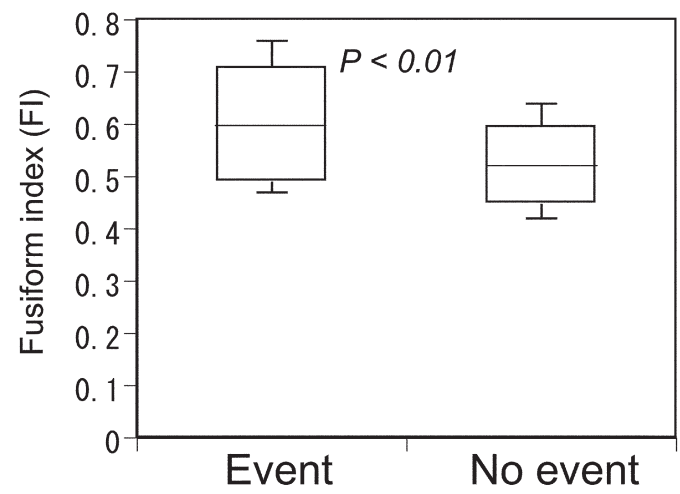

(B)

$(\%)$

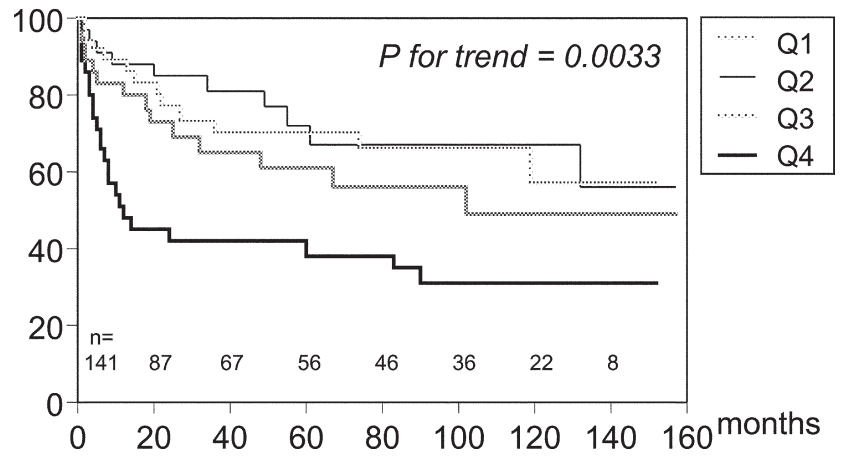

Figure 2. FI and late aortic events. A, Comparison of the FI between patients with and without late aortic events: Levels of FI are demonstrated by the box showing the 25th to 75th percentiles containing the median line and the lines showing the 10th to 90th percentiles. B, Kaplan-Meier event-free curves for late aortic events according to quartiles of the FI. Q, Quartile of FI: 01 $(<0.49), 02$ ( $\leq 0.49,<0.55), 03(\leq 0.55,<0.64), 04(\leq 0.64)$.

4 groups according to the 25th, 50th, and 75th percentiles of the FI $(0.49,0.55$, and 0.64 , respectively). A Cox proportional hazards model was used to identify the predominant predictors of late aortic events throughout the entire follow-up period by stepwise multivariate analyses. A stepwise selection method was used with a significance level of .10 for entering a variable into the model and .05 for retaining an explanatory variable from the model. The values of actuarial freedom from late aortic events were computed by using the Kaplan-Meier technique, and event-free curves were compared using the log-rank test. Data analysis was performed with StatView 5.0 for Windows (SAS Institute Inc, Cary, NC).

\section{Results}

Of the 141 patients, 59 (42\%) met the criteria for the development of late aortic events during the chronic phase. The mean duration between the onset of AAD and the most recent CT scan was 55.7 months and 49.3 months in the patient groups with and without late aortic events, respectively. The follow-up data were $98 \%$ complete, and the mean follow-up period was 64.4 months (range, 1-157 months). There was no significant difference in the mean follow-up periods between the 2 groups (68.1 vs 61.7 months).

In the 59 patients who met the criteria for late aortic events, the follow-up period from the onset of AAD to the development of the events was 1 to 132 months. Of these patients, $39(67 \%)$ had a maximum aortic diameter of 60 $\mathrm{mm}$ or more, $4(7 \%)$ had aortic rupture, $2(3 \%)$ had refractory pain, $2(3 \%)$ had visceral organ ischemia, $9(15 \%)$ had rapid aortic enlargement, and $3(5 \%)$ had rapid enlargement of ULPs. Of the 59 patients, 54 (92\%) underwent surgery; 3 $(5 \%)$ did not undergo surgery because of poor general condition, and $2(3 \%)$ refused surgery. Of the 54 patients who underwent surgery, $5(9 \%)$ died after the first surgery in the chronic phase ( 2 died of hemorrhage, 2 died of acute renal failure, and 1 died of bronchial occlusion resulting from hemorrhage from the aortopulmonary fistula). Two patients (4\%) died of aortic rupture at 24 and 66 months after the first operation. Four patients (7\%) died of nonaortic events ( 2 died of cancer, 1 died of stroke, and 1 committed suicide) after the first surgery. Of the 89 patients who did not meet the criteria for the late aortic events, $13(15 \%)$ died during the follow-up period ( 2 died of rupture of the dissected aorta, 6 died of cancer, 3 died of heart failure, 1 died of pneumonia, and 1 died of sudden death of unknown cause). Three patients (4\%) requested and underwent surgery before the development of late aortic events.

Figure 2, A shows a comparison between the FIs of the patients with and without late aortic events. The FI of patients with late aortic events (median value, 0.59) was significantly higher than that of patients without late aortic events (median value $0.53, P<.01$ ). The patients were divided into 4 groups according to the 25th, 50th, and 75th percentiles of the FI $(0.49,0.55$, and 0.64 , respectively). Figure 2, $B$ shows the event-free curves for late aortic events according to the quartiles of the FIs. Patients with the highest quartile had a significantly higher prevalence of late aortic events during the entire follow-up period ( $P$ for trend $=.0033$ ). Therefore, we used the highest quartile (FI $\geq$ 0.64 ) as a predictor of late aortic events for univariate and multivariate analyses.

Univariate variables that were significantly associated with late aortic events included ischemic heart disease $(P=$ .036), cerebrovascular disease $(P=.043)$, a patent false lumen $(P=.0018)$, a maximum aortic diameter of $40 \mathrm{~mm}$ or more $(P<.001)$, and an FI of 0.64 or more $(P<.001)$ (Table 3). All significant univariate variables were entered into the Cox proportional hazards model. A maximum aortic diameter of $40 \mathrm{~mm}$ or more $(P<.01)$, a patent false lumen $(P=.024)$, and an FI of 0.64 or more $(P=.013)$ 
TABLE 3. Statistical analysis of predictors for late aortic events

\begin{tabular}{lccccc}
\hline & \multicolumn{2}{c}{ Univariate } & & \multicolumn{2}{c}{ Multivariate } \\
\cline { 2 - 3 } \cline { 5 - 6 } Predictive factor & & & & Hazard Ratio \\
Sex & $\chi^{2}$ & $\boldsymbol{P}$ & $\boldsymbol{P}$ & $(95 \%$ CI) \\
Age $\geq 70$ y & 0.27 & .60 & & \\
Hypertension & 0.66 & .42 & & \\
Diabetes mellitus & 1.83 & .18 & & \\
Ischemic heart disease & 2.40 & .12 & & \\
Cerebrovascular disease & 4.38 & .036 & .45 & 1.67 (0.96-2.81) \\
COPD & 4.10 & .043 & .63 & 1.53 (0.78-2.67) \\
Hemodialysis & 0.61 & .44 & & \\
LVEF $\geq 70 \%$ & 1.46 & .23 & & \\
Type (DeBakey IIla/b) & 2.61 & .11 & & \\
Patent false lumen & 0.19 & .66 & & \\
Aortic diameter $\geq 40 \mathrm{~mm}$ & 9.70 & .0018 & .024 & 2.64 (1.62-4.03) \\
FI $\geq 0.64$ & 15.2 & $<.001$ & $<.01$ & 3.18 (2.12-5.05) \\
Mean systolic blood & 10.9 & $<.001$ & .013 & 2.73 (1.85-4.60) \\
$\quad$ pressure during follow- & 1.75 & .19 & & \\
$\quad$ up ( $\geq 140$ mm Hg) & & & & \\
\hline
\end{tabular}

$\overline{C l}$, Confidence interval; $C O P D$, chronic obstructive pulmonary disease; $L V E F$, left ventricular ejection fraction; $F I$, fusiform index.

were shown to be significant predictors of late aortic events. The hazard ratio for the presence of a maximum aortic diameter of $40 \mathrm{~mm}$ or more was 3.18 times higher than that for a maximum aortic diameter of less than $40 \mathrm{~mm}(95 \%$ confidence interval; range, 2.12-5.05); for a patent false lumen, this ratio was 2.64 times higher than that for a closed false lumen (95\% confidence interval; range 1.62-4.03). The hazard ratio for an FI of 0.64 or more was 2.73 times higher than that for an FI of less than 0.64 (95\% confidence interval; range 1.85-4.60). The values of actuarial freedom from late aortic events for patients with 1 of these predictors (Figure 3, A) were as follows. At 0.5, 1, 5, and 10 years, these values were $81 \%, 77 \%, 61 \%$, and $41 \%$, respectively, for patients with a patent false lumen (group P); $84 \%, 74 \%$, $46 \%$, and $40 \%$, respectively, for patients with a maximum aortic diameter of $40 \mathrm{~mm}$ or more (group $\mathrm{M}$ ); and $78 \%$, $44 \%, 44 \%$, and $44 \%$, respectively, for patients with an FI of 0.64 or more (group F). These curves were not significantly different from each other $(P=.72)$.

To obtain a more accurate estimation of the late aortic events, we analyzed the event-free curves for the following groups: patents with a maximum aortic diameter of $40 \mathrm{~mm}$ or more with a concomitant patent false lumen (group MP), patients with a maximum aortic diameter of $40 \mathrm{~mm}$ or more with an FI of 0.64 or more (group MF), and patients with a patent false lumen with an FI of 0.64 or more (group PF). The values of actuarial freedom from the late aortic events at $0.5,1,5$, and 10 years were $65 \%, 51 \%, 31 \%$, and $21 \%$, respectively, for group MP; 52\%, 33\%, 21\%, and $14 \%$,
(A)

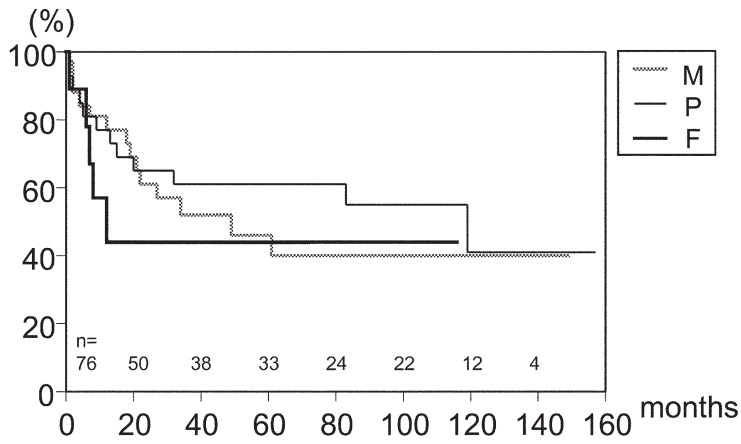

(B)

(\%)

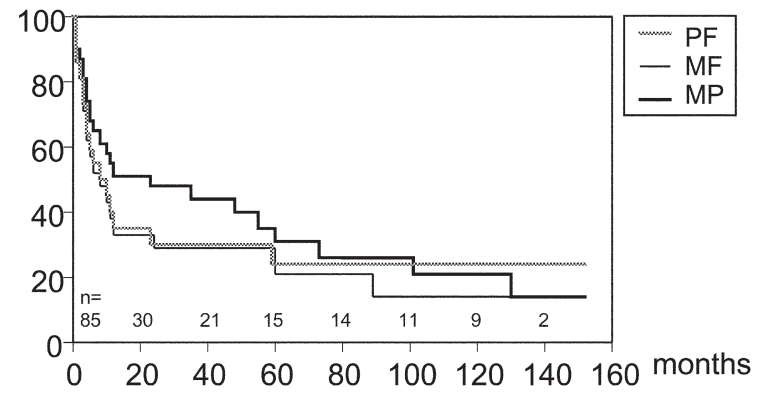

(C)

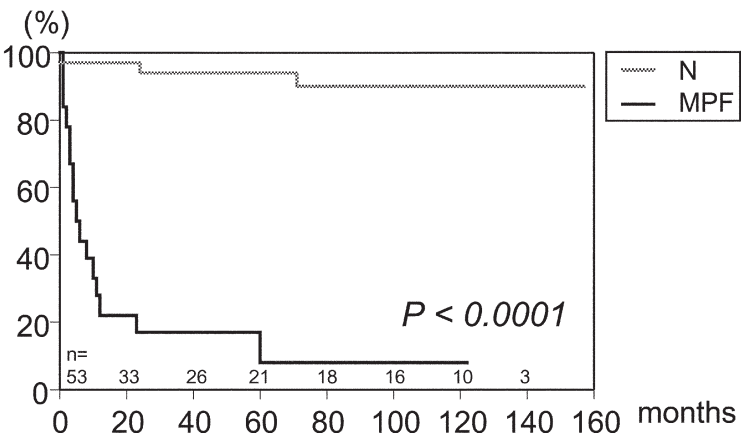

Figure 3. Kaplan-Meier event-free curves for late aortic events. A, Event-free curves of patients with 1 of the predominant predictors. M: Patients with a maximum aortic diameter of $\geq \mathbf{4 0} \mathrm{mm}$. P: Patients with a patent false lumen. F: Patients with an Fl of $\geq$ 0.64. B, Event-free curves of patients with 2 predominant predictors. MP: Patients with a maximum aortic diameter of $\geq \mathbf{4 0} \mathrm{mm}$ and a patent false lumen. MF: Patents with a maximum aortic diameter of $\geq 40 \mathrm{~mm}$ and an Fl of $\geq 0.64$. PF: Patients with a patent false lumen and an $\mathrm{Fl}$ of $\geq 0.64$. C, Event-free curves of patients with all 3 or none of the predominant predictors. $\mathbf{N}$ : Patients with none of the 3 predictors. MPF: Patients with all 3 predictors (a maximum aortic diameter of $\geq \mathbf{4 0} \mathrm{mm}$, a patent false lumen, and an $\mathrm{FI}$ of $\geq 0.64$ ).

respectively, for group MF; and 55\%, 35\%, 24\%, and 24\%, respectively, for group PF (Figure 3,B). The values of actuarial freedom from late aortic events for patients with all 3 predictors (group MPF) at $0.5,1,5$, and 10 years were $44 \%, 22 \%, 17 \%$, and $8 \%$, respectively, whereas those for 
(A)

(\%)

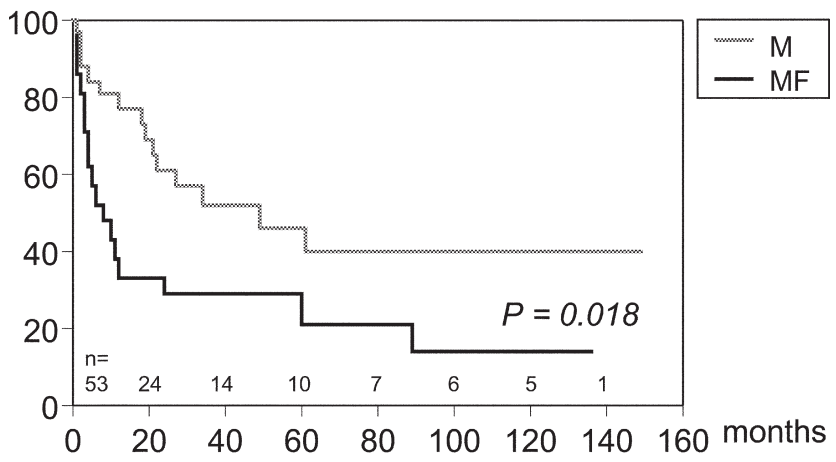

(B)

(\%)

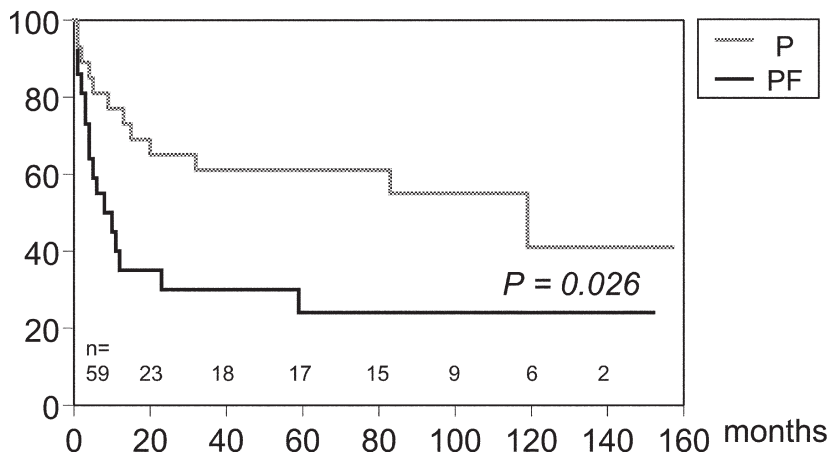

Figure 4. Comparison of the event-free curves between patients with 1 and 2 predictors. A, M: Patients with a maximum aortic diameter of $\geq \mathbf{4 0} \mathrm{mm}$. MF: Patents with a maximum aortic diameter of $\geq 40 \mathrm{~mm}$ and an Fl of $\geq 0.64$. B, P: Patients with a patent false lumen. PF: Patients with a patent false lumen and an $\mathrm{FI}$ of $\geq \mathbf{0 . 6 4}$.

the patients with none of these predictors (group N) were 97\%, 97\%, 94\%, and 90\%, respectively (Figure 3, C).

\section{Discussion}

The optimal indication for and timing of surgery in patients with Stanford type B AAD without complications have been controversial. We have suggested 2 therapeutic options for such patients that might provide better long-term results: Patients with predictors of late aortic events should undergo early surgery, whereas those without these predictors should continue to receive medical treatment. ${ }^{12}$

In the present study, the results indicated that late aortic events in type B AAD can be predicted by 3 independent acute-phase factors: a maximum diameter of the dissected aorta of $40 \mathrm{~mm}$ or more, a patent false lumen, and an FI of 0.64 or more. Patients with even 1 of these predictors should be recommended early interventions (surgery or stent-graft implantation) to prevent the development of late aortic events. However, the event-free rates of patients with 1 of these predictors at 10 years were approximately $40 \%$ to 50\%; this indicated that approximately half of the patients can be treated medically on a long-term basis (Figure 3, A). After careful observation, we concluded that patients with 1 of these predictors can be treated medically as long as the aortic diameter does not increase.

The presence of more than 1 of these predictors in a patient may be regarded as a more reliable indication for surgical intervention. Other studies, in addition to our study, reported that a maximum aortic diameter of $40 \mathrm{~mm}$ or more and a patent false lumen are the predominant predictors of late aortic events. ${ }^{6-12}$ In the present study, the event-free curves of patients with a maximum aortic diameter of 40 $\mathrm{mm}$ or more and an FI of 0.64 or more (group MF) were significantly different from those of patients with a maximum aortic diameter of $40 \mathrm{~mm}$ or more alone (group M) (Figure 4, A, $P=.018$ ). Similarly, the event-free curves of patients with a patent false lumen and an FI of 0.64 or more (group PF) was also significantly different from those of patients with a patent false lumen alone (group P) (Figure 4, $B, P=.026)$. Therefore, a combination of the previously known predictors with an FI of 0.64 or more may aid in predicting late aortic events more accurately. We recommend early interventions or at least close follow-up for patients with 2 of these predictors. Most important, the event-free rates of patients with all 3 predictors (group MPF) at 1, 5, and 10 years were $22 \%, 17 \%$, and $8 \%$, respectively; these values were considerably lower than those of the MP, MF, and PF groups. In view of this, we suggest that patients with all 3 predominant predictors should be recommended surgical interventions during the subacute or early chronic phase before late aortic events can develop.

On the other hand, most patients without predictors (group N) showed good long-term results because the dissected aorta showed a natural course of recovery (Figure 3, $C)$. The event-free rates of group $\mathrm{N}(\mathrm{n}=35)$ at 1,5 , and 10 years $(97 \%, 94 \%$, and $90 \%$, respectively) were satisfactory. None of the patients in this group died of aortic events, and 12 patients (34\%) showed a reduction in the aortic diameter. Therefore, patients who are expected to have a lower probability of developing late aortic events should not be recommended surgery during the acute phase and should continue to receive medical treatment.

A higher FI may predict earlier aortic dilatation. The event-free rate at 1 year for group F (44\%) was considerably lower than that for group P (77\%) or group M (74\%), whereas the event-free rate of group $\mathrm{F}$ at 10 years (44\%) was close to that of group P (40\%) or group M (41\%). The event-free curves of these groups $(\mathrm{P}, \mathrm{M}$, and $\mathrm{F})$ were not significantly different from each other (Figure $3, A$ ). These 
results indicate that the expansion of the aortic diameter in patients with a higher FI may be faster than that in patients with a lower FI, although the number of patients who developed late aortic events on a long-term basis was equal in both groups.

In the present study, we focused on the degree of fusiform dilatation of the proximal descending aorta as a predictor of late aortic events in patients with type B AAD. The initial change in the formation of an aneurysm is structural and results from a degenerative process in the vessel wall. ${ }^{14}$ Once the morphologic change has occurred, the blood flow pattern is likely to change, thereby leading to alterations in the shear stresses and consequent strain; this accelerates the development of the aneurysm so that a vicious circle is created. The geometry of the aorta is altered during aneurysmal degeneration, and the geometric relationship between the transverse and distal segment of the aortic arch becomes increasingly distorted during the process of development of a distal arch aortic aneurysm. ${ }^{13,14,16}$ In a fusiform dilatation of the proximal descending aorta, the magnitude of the initial morphologic change may be much more than that in a non-fusiform proximal descending aorta; this worsens the disturbed flow pattern and may result in accelerating the dilatation of the proximal descending aorta. The outer curvature of the dissected aortic wall, which often consists of adventitia and dissected media, was thin and fragile and may also accelerate the dilatation of the aorta. We did not use the location of the entry site as a predictor because the location of the entry sites could not be accurately confirmed in any patient. However, in most of the patients with a high FI who developed late aortic events, the entry site was located at the distal arch. This may support the hypothesis that the flow-pattern disturbances in the proximal descending aorta directly lead to aortic dilatation.

There are some limitations to the present study. First, there were difficulties in obtaining accurate measurements of the aortic diameter on axial-CT images. We used 3dimensional CT to determine the dimensions of the segment of the aorta under consideration. However, in patients in whom the 3-dimensional CT data were unavailable, we used a measurement method with axial-CT scan reports similar to the method used in several studies. ${ }^{10,15}$ In addition, we did not consider the laterality of the aortic diameters. The measurements used in the present study may have underestimated the aortic diameter in some cases. Second, other factors such as blood-flow velocity and flow-pattern disturbances in the proximal descending aorta, which may influence the development of late aortic events, were not evaluated in the present study. Finally, the recent advent of endovascular-stent grafting may change the therapeutic strategies for type B AAD. ${ }^{3,5,17-27}$

In summary, a maximum aortic diameter of $40 \mathrm{~mm}$ or more, a patent false lumen, and an FI of 0.64 or more during the acute phase of type B AAD without complications were predominant independent predictors of the late aortic events associated with this disorder. A higher FI value might help to predict the late aortic events earlier. Patients without these predictors should continue to receive conventional medical treatment because they are predicted to have a satisfactory natural course of recovery of the dissected aorta. On the contrary, patients with 1 of these predictors can be treated medically but with close follow-up. Patients with 2 of the predominant predictors should be considered for early intervention (surgery or stent-graft implantation) or at least close follow-up. Patients with all 3 predictors are strongly predicted to have a high incidence of developing late aortic events; therefore, they should undergo the intervention during the subacute or early chronic phase before late aortic events can develop.

\section{Conclusions}

The degree of fusiform dilatation of the proximal descending aorta in patients with Stanford type B AAD without complications can be used to predict late aortic adverse events. This predictor may be useful for developing safer and less-invasive treatment strategies for type B AAD without complications.

We thank Professor Masashi Komeda (Department of Cardiovascular Surgery, Kyoto University) for helpful suggestions and comments, and Masaya Yamamoto (The Institute for Frontier Medical Sciences, Kyoto University) for statistical collaboration in this article.

\section{References}

1. Myrmel T, Lai DT, Miller DC. Can the principles of evidence-based medicine be applied to the treatment of aortic dissections? Eur J Cardiothorac Surg. 2004;25:236-42.

2. Hagan PG, Nienaber CA, Isselbacher EM, et al. The International Registry of Acute Aortic Dissection (IRAD): new insights into an old disease. JAMA. 2000;283:897-903.

3. Umana JP, Lai DT, Mitchell RS, et al. Is medical therapy still the optimal treatment strategy for patients with acute type B aortic dissections? J Thorac Cardiovasc Surg. 2002;124:896-910.

4. Safi HJ, Estrera AL. Aortic dissection. Br J Surg. 2004;91:523-5.

5. Genoni M, Paul M, Tavakoli R, et al. Predictors of complications in acute type B aortic dissection. Eur J Cardiothorac Surg. 2002;22:59-63.

6. Masuda Y, Yamada Z, Morooka N, Watanabe S, Inagaki Y. Prognosis of patients medically treated aortic dissection. Circulation. 1991; 84[suppl III]:III-7-13.

7. Juvonen T, Ergin MA, Galla JD, et al. Risk factors for rupture of chronic type B dissections. J Thorac Cardiovasc Surg.1999;117:776-86.

8. Kato M, Bai H, Sato K, et al. Determining surgical indications for acute type B dissection based on enlargement of aortic diameter during the chronic phase. Circulation. 1995;92[Suppl II]:II-107-12.

9. Bernard Y, Zimmermann H, Chocron S, et al. False lumen patency as a predictor of late outcome in aortic dissection. Am J Cardiol. 2001; $87: 1378-82$

10. Sueyoshi E, Sakamoto I, Hayashi K, Yamaguchi T, Imada T. Growth rate of aortic diameter in patients with type $\mathrm{B}$ aortic dissection during the chronic phase. Circulation. 2004;110[Suppl II]:II-256-61.

11. Hata M, Shiono M, Inoue T, et al. Optimal treatment of type B acute aortic dissection: long-term medical follow-up results. Ann Thorac Surg. 2003;75:1781-4. 
12. Marui A, Mochizuki T, Mitsui N, Koyama T, Kimura F, Horibe M. Toward the best treatment for uncomplicated patients with type B acute aortic dissection: a consideration for sound surgical indication. Circulation. 1999;100[Suppl II]:II-275-80.

13. Hardy CJ, Bolster BD Jr, McVeigh ER, Iben IE, Zerhouni EA. Pencil excitation with interleaved Fourier velocity encoding: NMR measurement of aortic distensibility. Magn Reson Med. 1996;35:814-9.

14. Bogren HG, Mohiaddin RH, Yang GZ, Kilner PJ, Firmin DN. Magnetic resonance velocity vector mapping of blood flow in thoracic aortic aneurysms and grafts. J Thorac Cardiovasc Surg. 1995;110:704-14.

15. Hirose $Y$, Hamada $S$, Takamiya M, Imakita $S$, Naito H, Nishimura $T$. Aortic aneurysms: growth rates measured with CT. Radiology. 1992; 185:249-52.

16. Kilner PJ, Yang GZ, Mohiaddin RH, Firmin DN, Longmore DB. Helical and retrograde secondary flow patterns in the aortic arch studied by three-directional magnetic resonance velocity mapping. Circulation. 1993;88:2235-47.

17. Dake MD, Kato N, Mitchell RS, et al. Endovascular stent-graft placement for the treatment of acute aortic dissection. $N$ Engl J Med. 1999;340:1546-52.

18. Kato N, Shimono T, Hirano T, et al. Midterm results of stent-graft repair of acute and chronic aortic dissection with descending tear: the complication-specific approach. J Thorac Cardiovasc Surg. 2002;124:306-12.

19. Chavan A, Lotz J, Oelert F, Galanski M, Haverich A, Karck M. Endoluminal treatment of aortic dissection. Eur Radiol. 2003;13:2521-34.
20. Appoo JJ, Moser WG, Fairman RM, et al. Thoracic aortic stent grafting: improving results with newer generation investigational devices. J Thorac Cardiovasc Surg. 2006;131:1087-94.

21. Doss M, Wood JP, Balzer J, Martens S, Deschka H, Moritz A. Emergency endovascular interventions for acute thoracic aortic rupture: four-year follow-up. J Thorac Cardiovasc Surg. 2005;129:645-51.

22. Gleason TG. Thoracic aortic stent grafting: is it ready for prime time? J Thorac Cardiovasc Surg. 2006;131:16-8.

23. Xu SD, Huang FJ, Yang JF, et al. Endovascular repair of acute type B aortic dissection: early and mid-term results. J Vasc Surg. 2006;43: 1090-5.

24. Bockler D, Schumacher H, Ganten M, et al. Complications after endovascular repair of acute symptomatic and chronic expanding Stanford type B aortic dissections. J Thorac Cardiovasc Surg. 2006; 132:361-8.

25. Schoder M, Grabenwoger M, Holzenbein T, et al. Endovascular repair of the thoracic aorta necessitating anchoring of the stent graft across the arch vessels. J Thorac Cardiovasc Surg. 2006;131:380-7.

26. Marcheix B, Dambrin C, Bolduc JP, et al. Midterm results of endovascular treatment of atherosclerotic aneurysms of the descending thoracic aorta. J Thorac Cardiovasc Surg. 2006;132:1030-6.

27. Iyer VS, Mackenzie KS, Tse LW, et al. Early outcomes after elective and emergent endovascular repair of the thoracic aorta. J Vasc Surg. 2006;43:677-83.

\section{The Journal of Thoracic and Cardiovascular Surgery Conflict of Interest Policy}

To assure fairness to authors submitting work for consideration in The Journal of Thoracic and Cardiovascular Surgery, a mechanism exists for managing conflicts of interest. The editor and each of the section editors complete a "Conflict of Interest" form that identifies any and all relationships with commercial and other academic entities. When the editor has a potential conflict because of a relationship with another entity or author, the editor appoints an alternate editor from among the section editors or editorial board members who assumes the entire responsibility for final decisions on the manuscript in question. The editor does not read the reviews that are submitted nor engage in discussing the manuscript prior to the final decision. When the conflict of interest involves a section editor, a "guest section editor" is appointed who fills the role normally played by the conflicted section editor. All members of the editorial board and reviewers are asked to indicate any conflict of interest when they agree to review a manuscript. 